\title{
Hopf Bifurcation and Chaos in Synchronous Reluctance Motor Drives
}

\author{
Y. Gao, Student Member, IEEE, and K. T. Chau, Senior Member, IEEE
}

\begin{abstract}
This paper first presents the occurrence of Hopf bifurcation and chaos in a practical synchronous reluctance motor drive system. Based on the derived nonlinear system equation, the bifurcation analysis shows that the system loses stability via Hopf bifurcation when the $d$-axis component of its three-phase motor voltages loses its control. Moreover, the corresponding Lyapunov exponent calculation further proves the existence of chaos. Finally, computer simulations and experimental results are used to support the theoretical analysis.
\end{abstract}

Index Terms-Bifurcation, chaos, motor drives, synchronous reluctance motors.

\section{INTRODUCTION}

W ITH ever-increasing concern for our environment, the development of motor drives has focused on pursuing higher efficiency and better recyclability. Among those modern motor drives, including the induction motor (IM), permanent-magnet brushless motor (PMBM), and variable-reluctance motor (VRM), the VRM class takes the advantages of simple and rugged structure, good compatibility with the power converter, and high recyclability for the core and winding. The VRM is further divided into the synchronous reluctance motor (SynRM) and switched reluctance motor (SRM). The SynRM adopts a distributed winding and sinusoidal wave, whereas the SRM uses a concentrated winding and rectangular wave. So, the SynRM can essentially eliminate the torque pulsation and acoustic noise problems that are intractable in the SRM. Increasingly, it can offer the capability of electrical fault tolerance, such as one-phase open circuit, one phase short circuit, and phase-to-phase short circuit. With the use of field-oriented control or vector control, the SynRM drive can compete favorably with the IM drive for high-performance drive applications such as electric vehicle propulsion [1]-[5].

Starting from the late 1980 s, chaos has been identified to be a real phenomenon in power electronics. Then, many investigations into chaotic behavior of dc-dc converters were conducted in the 1990s [6]-[9]. However, the investigation of chaos in motor drives has been surprisingly rare. Namely, the bifurcation and chaos in an IM drive were investigated in [10], the strange attractor and chaos in a PMBM drive were studied in [11], bifurcation and chaos in a permanent-magnet synchronous motor (PMSM) were simulated in [12], the chaos in voltage-mode and current-mode dc motor drives were analyzed in [13] and [14],

Manuscript received February 20, 2003. This work was supported by a grant from the Research Grants Council of Hong Kong Special Administrative Region, China.

The authors are with the Department of Electrical and Electronic Engineering, The University of Hong Kong, Hong Kong, China.

Digital Object Identifier 10.1109/TEC.2004.827012

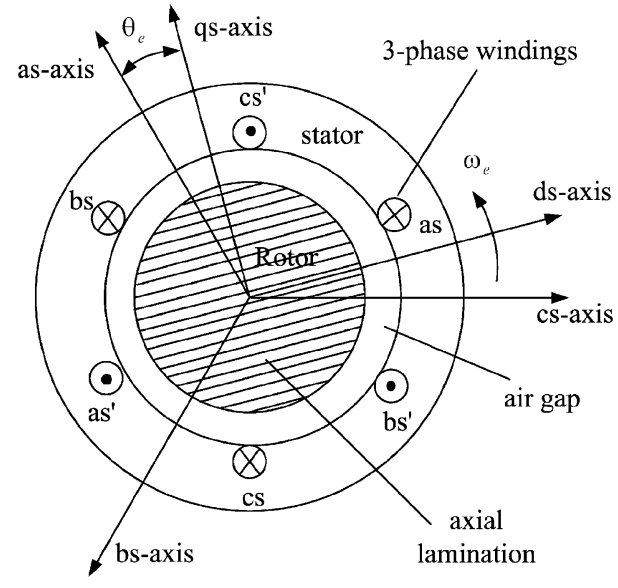

Fig. 1. Schematic diagram of three-phase SynRM

as well as the subharmonics and chaos in a SRM drive were discussed in [15]. To the best of authors' knowledge, the investigation of chaos in the SynRM drive was absent in the literature.

The purpose of this paper is to first analyze the bifurcation and chaotic behavior of SynRM drives. A practical SynRM drive adopting field-oriented control will be used for exemplification. Bifurcation analysis and Lyapunov exponent calculation will be employed to investigate the corresponding chaotic behavior. Various computer simulations and experiments will be carried out to support the theoretical results.

In Section II, a brief introduction of the SynRM drive, including its configuration, mathematical model, and operating principle will be given. In Section III, the SynRM drive adopting field-oriented control will be modeled. Based on this newly derived model, bifurcation and chaotic analyses will be conducted in Sections IV and V, respectively. Finally, simulation and experimental results will be given to verify the theoretical prediction in Section VI.

\section{SYNRM DRIVE}

The SynRM is a singly salient machine. Its stator is typically equipped with three-phase sinusoidally distributed windings, which is similar to that of an IM. Its rotor is purposely constructed with salient poles so as to produce the desired reluctance torque for electromechanical energy conversion. This salient rotor can be derived by using the geometrically salient-pole structure, the axially laminated structure, or the flux-barrier structure. The higher the saliency ratio, the larger the reluctance torque can be produced.

Fig. 1 shows a three-phase two-pole SynRM with an axially laminated rotor structure. Since both the field winding and 


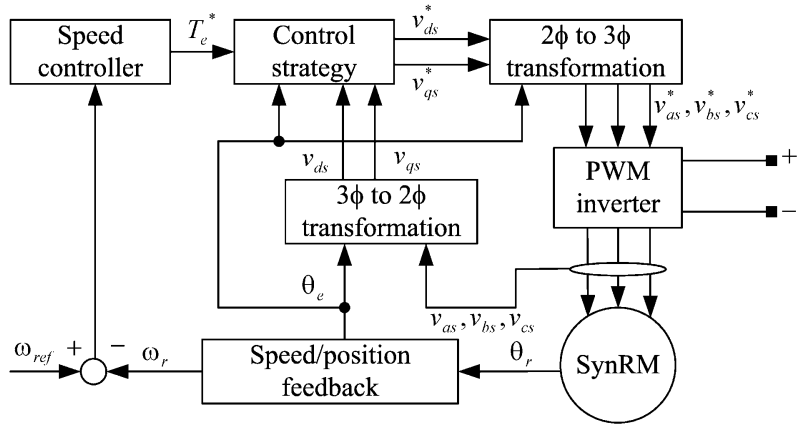

Fig. 2. Control diagram of three-phase SynRM drive.

damper winding are absent in the SynRM, the corresponding system equations are given by

$$
\left\{\begin{array}{l}
v_{a s}=R_{s} i_{a s}+\frac{d \psi_{a s}}{d t} \\
v_{b s}=R_{s} i_{b s}+\frac{d \psi_{b s}}{d t} \\
v_{c s}=R_{s} i_{c s}+\frac{d \psi_{c s}}{d t}
\end{array}\right.
$$

where $v_{a s}, v_{b s}$, and $v_{c s}$ are the stator phase voltages; $i_{a s}, i_{b s}$, and $i_{c s}$ are the stator phase currents; $\psi_{a s}, \psi_{b s}$, and $\psi_{c s}$ are the stator flux linkages; and $R_{s}$ is the stator resistance per phase.

By applying the well-known Park's transformation, the system equations given by (1) can be rewritten as [16], [17]

$$
\left\{\begin{array}{l}
v_{d s}=R_{s} i_{d s}+\frac{d \psi_{d s}}{d t}-\omega_{e} L_{q s} i_{q s} \\
v_{q s}=R_{s} i_{q s}+\frac{d \psi_{q s}}{d t}+\omega_{e} L_{d s} i_{d s} \\
\psi_{d s}=L_{m d} i_{d s}+L_{l s} i_{d s}=L_{d s} i_{d s} \\
\psi_{q s}=L_{m q} i_{q s}+L_{l s} i_{q s}=L_{q s} i_{q s}
\end{array}\right.
$$

where the subscripts $d s$ and $q s$ represent the corresponding direct-axis ( $d$-axis) and quadrature-axis ( $q$-axis) quantities, respectively, $L_{m d}$ and $L_{m q}$ are the magnetizing inductances, $L_{l s}$ is the stator leakage inductance, and $\omega_{e}$ is the electrical rotor speed. Hence, the electromagnetic torque is expressed as

$$
T_{e}=\frac{3}{2} \frac{P}{2}\left(\psi_{d s} i_{q s}-\psi_{q s} i_{d s}\right)
$$

where $P$ is the number of poles. In terms of $L_{d s}$ and $L_{q s}$, it can be rewritten as

$$
T_{e}=\frac{3}{2} \frac{P}{2}\left(L_{d s}-L_{q s}\right) i_{d s} i_{q s}
$$

From (4), it can be found that the higher the saliency $\left(L_{d s} / L_{q s}\right)$ of the SynRM, the greater the difference between $L_{d s}$ and $L_{q s}$, the larger the value of $T_{e}$ is produced. Then, the motion equation is given by

$$
J \frac{d \omega_{r}}{d t}=T_{e}-B \omega_{r}-T_{L}
$$

where $J$ is the moment of inertia of the drive system, $B$ is the viscous friction coefficient, $T_{L}$ is the load torque, and $\omega_{r}=$ $2 \omega_{e} / P$ is the mechanical rotor speed.

Based on the above $d-q$ model, a modern SynRM drive generally employs field-oriented control as illustrated in Fig. 2.

\section{SySTEM MODELING}

In order to study the behavior of the SynRM drive, the system dynamical model can be deduced from (2), (4), and (5) as given by

$$
\left\{\begin{array}{l}
v_{d s}=L_{d s} \frac{d i_{d s}}{d t}+R_{s} i_{d s}-\omega_{e} L_{q s} i_{q s} \\
v_{q s}=L_{q s} \frac{d i_{q s}}{d t}+R_{s} i_{q s}+\omega_{e} L_{d s} i_{d s} \\
J \frac{d \omega_{r}}{d t}=\frac{3}{2} \frac{P}{2}\left(L_{d s}-L_{q s}\right) i_{d s} i_{q s}-B \omega_{r}-T_{L}
\end{array}\right.
$$

The key merit of field-oriented control is to decouple the field component ( $d$-axis stator current $\left.i_{d s}\right)$ and the torque component ( $q$-axis stator current $i_{q s}$ ) for high-performance operation. In general, the SynRM is maintained fully fluxed by exciting it with a constant $i_{d s}$ while the torque is regulated by controlling $i_{q s}$. Based on a voltage-fed inverter, $i_{d s}$ and $i_{q s}$ are governed by $v_{d s}$ and $v_{q s}$, respectively. The constant value of $v_{d s}$ can be easily deduced by substituting $d i_{d s} / d t=0$ into (6), whereas $v_{q s}$ is regulated by the speed error using PID control. The corresponding control criteria are set below

$$
\left\{\begin{array}{l}
v_{d s}=R_{s} i_{d s}-\omega_{e} L_{q s} i_{q s} \\
v_{q s}=\left(k_{p}+\frac{k_{i}}{s}+s k_{d}\right)\left(\omega_{r}-\omega_{\mathrm{ref}}\right)
\end{array}\right.
$$

where $\omega_{\text {ref }}$ is the reference mechanical rotor speed.

The above field-oriented control works well provided that $i_{d s}$ can be kept constant and under proper control. In case $v_{d s}$ loses its control, the SynRM drive may exhibit strange behavior. For the sake of simplicity, the analytical derivation is based on the assumption that $v_{d s}=0$ and $k_{i}=k_{d}=0$ which may be somewhat unrealistic. Otherwise, the analytical solution cannot be derived and only the numerical results can be generated.

Thus, based on (6) and (7), the SynRM drive can be expressed as

$$
\left\{\begin{array}{l}
v_{d s}=L_{d s} \frac{d i_{d s}}{d t}+R_{s} i_{d s}-\omega_{e} L_{q s} i_{q s} \\
k_{p}\left(\omega_{r}-\omega_{r e f}\right)=L_{q s} \frac{d i_{q s}}{d t}+R_{s} i_{q s}+\omega_{e} L_{d s} i_{d s} \\
J \frac{d \omega_{r}}{d t}=\frac{3}{2} \frac{P}{2}\left(L_{d s}-L_{q s}\right) i_{d s} i_{q s}-B \omega_{r}-T_{L}
\end{array}\right.
$$

Rearranging $i_{d s}, i_{q s}$, and $\omega_{r}$ as the state variables, (8) can be rewritten as

$$
\left\{\begin{aligned}
\frac{d i_{d s}}{d t} & =\frac{\left(v_{d s}-R_{s} i_{d s}+\omega_{e} L_{q s} i_{q s}\right)}{L_{d s}} \\
\frac{d i_{q s}}{d t} & =\frac{\left[-R i_{q s}-\omega_{e} L_{d s} i_{d s}+k_{p}\left(\omega_{r}-\omega_{r e f}\right)\right]}{L_{q s}} \\
\frac{d \omega_{r}}{d t} & =\frac{\left[\frac{3}{2} \frac{P}{2}\left(L_{d s}-L_{q s}\right) i_{d s} i_{q s}-B \omega_{r}-T_{L}\right]}{J} .
\end{aligned}\right.
$$

It should be noted that the feedback effect $k_{p}\left(\omega_{r}-\omega_{r e f}\right)$ has been taken into consideration which is absent in the open-loop cases in [11] and [12].

The system equation can be further simplified by transforming $t$ to $\tau t^{\prime}$ and $\left(i_{d s}, i_{q s}, \omega_{e}\right)$ to $(x, y, z)$ as defined by [11] and [12]

$$
\left[\begin{array}{c}
i_{d s} \\
i_{q s} \\
\omega_{e}
\end{array}\right]=\left[\begin{array}{ccc}
b k & 0 & 0 \\
0 & k & 0 \\
0 & 0 & \frac{1}{\tau}
\end{array}\right]\left[\begin{array}{l}
x \\
y \\
z
\end{array}\right]
$$


where $b=L_{q s} / L_{d s}, k=\sqrt{8 J / 3 P^{2}\left(L_{d s}-L_{q s}\right) b \tau^{2}}$, and $\tau=L_{q s} / R_{s}$. Hence, the SynRM drive can be expressed as

$$
\left\{\begin{array}{l}
\frac{d x}{d t^{\prime}}=v_{d s}^{\prime}-b x+y z \\
\frac{d y}{d t^{\prime}}=-y-x z+c\left(z-z_{\mathrm{ref}}\right) \\
\frac{d z}{d t^{\prime}}=x y-a z+T_{L}^{\prime}
\end{array}\right.
$$

where $v_{d s}^{\prime}=v_{d s} / k R_{s}, c=2 k_{p} / k P L_{q s}, z_{\text {ref }}=\tau \omega_{\text {ref }}, a=$ $B L_{q s} / J R_{s}$, and $T_{L}^{\prime}=P \tau^{2} T_{L} / 2 J$.

\section{LOCAL STABILITY AND HOPF BIFURCATION}

It is essential to discuss the stability of the equilibrium point and the trajectory around the equilibrium point by deriving the eigenvalues of the system at the equilibrium point.

\section{A. Special Case}

For the special case when $v_{d s}=0, \omega_{\text {ref }}=0$, and $T_{L}=0$, the system (11) becomes

$$
\left\{\begin{array}{l}
\frac{d x}{d t^{\prime}}=-b x+y z \\
\frac{d y}{d t^{\prime}}=-y-x z+c z \\
\frac{d z}{d t^{\prime}}=x y-a z .
\end{array}\right.
$$

The equilibrium point can be deduced by setting the derivatives in (12) equal to zero. Obviously, the origin is a trivial equilibrium point. The nonzero equilibria can be solved by the following equations:

$$
\left\{\begin{array}{l}
x^{2}-c x+a=0 \\
y^{2}-a b=0 \\
a z-x y=0 .
\end{array}\right.
$$

Considering $i_{d s}, i_{q s}, \omega_{r}$ and their transformed quantities $x$, $y, z$ are all realistic parameters, there are three possible cases:

1) if $\Delta=c^{2}-4 a<0$, one equilibrium point $(0,0,0)$;

2) if $\Delta=c^{2}-4 a=0$, three equilibrium points $(0,0,0)$ and $(c / 2, \pm \sqrt{a b}, \pm \sqrt{b / a})$

$3)$ if $\Delta=c^{2}-4 a>0$, five equilibrium points $(0,0,0)$ and $\left(\left(c \pm \sqrt{c^{2}-4 a}\right) / 2, \pm \sqrt{a b}, x_{e q} y_{e q} / a\right)$

The local stability of the equilibrium point is described by the eigenvalues of the system characteristic

$$
\operatorname{det}(\lambda \mathbf{I}-\mathbf{J})=0
$$

where $\lambda$ is the eigenvalue, $\mathbf{I}$ is the identity matrix, and $\mathbf{J}$ is the Jacobian matrix of the transformed system evaluated at the equilibrium point $\left(x_{e q}, y_{e q}, z_{e q}\right)$. Hence, the eigenvalues can be deduced from an explicit cubic equation as given by

$$
\lambda^{3}+r_{2} \lambda^{2}+r_{1} \lambda+r_{0}=0 .
$$

It is easy to check that the origin is a locally stable equilibrium point since the corresponding eigenvalues are all negative, being $-a,-b$, and -1 .

For the nonzero equilibrium case, an explicit form of (15) can be obtained using (13)

$$
\lambda^{3}+(a+b+1) \lambda^{2}+\frac{b\left(a+x_{\mathrm{eq}}^{2}\right) \lambda}{a}+2 b\left(x_{\mathrm{eq}}^{2}-a\right)=0 .
$$

TABLE I

ROUTH-HURWITZ ARRAY FOR LOCAL STABILITY

\begin{tabular}{ccc}
\hline$s^{3}$ & 1 & $b\left(a+x_{e q}{ }^{2}\right) / a$ \\
$s^{2}$ & $a+b+1$ & $2 b\left(x_{e q}{ }^{2}-a\right)$ \\
$s^{1}$ & $\frac{b\left(x_{e q}{ }^{2}+a\right)}{a}-\frac{2 b\left(x_{e q}{ }^{2}-a\right)}{a+b+1}$ & 0 \\
$s^{0}$ & $2 b\left(x_{e q}{ }^{2}-a\right)$ & 0 \\
\hline
\end{tabular}

Applying the Routh-Hurwitz stability criterion, as shown in Table I, the local stability is guaranteed by the following condition:

$$
\left\{\begin{array}{l}
2 b\left(x_{\mathrm{eq}}^{2}-a\right)>0 \\
\frac{a b(3 a+b+1)-b(a-b-1) x_{\mathrm{eq}}^{2}}{a(a+b+1)}>0 .
\end{array}\right.
$$

According to [18], the system will exhibit Hopf bifurcation if there exists a pair of complex eigenvalues satisfying the following criteria:

$$
\left\{\begin{array}{l}
\left.\operatorname{Re}(\lambda)\right|_{c=c_{0}}=0 \\
\left.\operatorname{Im}(\lambda)\right|_{c=c_{0}} \neq 0 \\
\left.\frac{d}{d c} \operatorname{Re}(\lambda)\right|_{c=c_{0}} \neq 0
\end{array}\right.
$$

where $c_{0}$ is the critical value of $c$ at which Hopf bifurcation occurs.

Considering that there exists a pure imaginary nonzero characteristic root $\lambda=n j(n \neq 0)$ in (16), it yields

$$
-n^{3} j-(a+b+1) n^{2}+\frac{b}{a}\left(a+x_{\mathrm{eq}}^{2}\right) n j+2 b\left(x_{\mathrm{eq}}^{2}-a\right)=0 .
$$

By separating the real part and the imaginary part, it results

$$
\left\{\begin{array}{l}
n^{2}=\frac{b\left(a+x_{\mathrm{eq}}^{2}\right)}{a} \\
n^{2}=\frac{2 b\left(x_{\mathrm{eq}}^{2}-a\right)}{(a+b+1)} .
\end{array}\right.
$$

For positive $a, b$, and $c$, the existence of $n$ is guaranteed by the following condition:

$$
\left\{\begin{array}{l}
a-b-1>0 \\
x_{\mathrm{eq}}^{2}-a>0 \\
x_{\mathrm{eq}}^{2}=\frac{a(3 a+b+1)}{(a-b-1)} .
\end{array}\right.
$$

This condition shows that the system parameters $L_{d s}, L_{q s}, J$, and $B$ of the SynRM drive have a critical effect on the existence of bifurcation. If their values are not properly set, Hopf bifurcation and chaos may occur.

It is straightforward to check that $\left(\left(c-\sqrt{c^{2}-4 a}\right) / 2\right)^{2}<a$ for positive $a$ and $c$. For this reason, the equilibrium point pair $\left(\left(c-\sqrt{c^{2}-4 a}\right) / 2, \pm \sqrt{a b}, x_{\mathrm{eq}} y_{\mathrm{eq}} / a\right)$ if it exists, will not meet the bifurcation condition in (18). From (17), they are always locally unstable.

By substituting $x_{\text {eq }}=\left(c+\sqrt{c^{2}-4 a}\right) / 2$ into (21), the critical value of $c$ at which Hopf bifurcation occurs can be obtained as

$$
c_{0}=4 a \sqrt{\frac{a}{[(a-b-1)(3 a+b+1)]}} .
$$


TABLE II

RELATIONSHIP BETWEEN SIGNS OF LYAPUNOV EXPONENTS AND TYPE OF ATTRACTORS

\begin{tabular}{cc}
\hline Signs & Type of Attractors \\
\hline$(-,-,-)$ & Fixed point \\
$(0,-,-)$ & Limit cycle \\
$(0,0,-)$ & Quasi-periodic torus \\
$(+, 0,-)$ & Chaotic \\
\hline
\end{tabular}

The corresponding critical eigenvalues are given by

$$
\lambda_{1}=-(a+b+1) \quad \lambda_{2,3}= \pm j \sqrt{\frac{b c_{0}\left(c_{0}+\sqrt{c_{0}^{2}-4 a}\right)}{2 a}} .
$$

Obviously, from (17), when $c$ is large enough while still smaller than the critical value $c_{0}$, the equilibrium point pair $\left(\left(c+\sqrt{c^{2}-4 a}\right) / 2, \pm \sqrt{a b}, x_{\text {eq }} y_{\text {eq }} / a\right)$ is stable. Once the value of $c$ exceeds the critical value, it becomes unstable saddle points. Furthermore, it may even become chaotic attractors as shown in the coming sections.

\section{B. General Case}

In general, the solution of the system (11) will lead to a fifth-order polynomial. Consequently, the equilibrium point $\left(x_{\mathrm{eq}}, y_{\mathrm{eq}}, z_{\mathrm{eq}}\right)$ has five possible roots-five real roots, three real roots, plus a pair of complex conjugate roots, or one real root plus two pairs of complex conjugate roots. Analytical results for this case are almost impossible. Nevertheless, the numerical analysis can always be performed.

\section{From Bifurcation to ChaOs}

Under the occurrence of Hopf bifurcation, the dynamical system may demonstrate complicated behavior, that is, chaos. To further identify the chaotic behavior, the calculation of Lyapunov exponents plays an important role. Namely, a system will exhibit chaotic behavior if at least one of its Lyapunov exponents is positive [19]. For a three-dimensional (3-D) state-space system described by a set of three first-order differential equations, the corresponding type of attractors (fixed point, limit cycle, quasiperiod torus, and chaotic) can be directly determined by the signs of Lyapunov exponents [20] as listed in Table II. Obviously, the SynRM drive described by (11) belongs to the aforementioned 3-D state-space system. Thus, this SynRM drive will demonstrate chaotic behavior if its Lyapunov exponents are one zero, one positive, and one negative.

The notion of Lyapunov exponent is a generalization of the idea of an eigenvalue as a measure of the stability of a fixed point or a characteristic exponent as the measure of the stability of a periodic orbit. For a chaotic trajectory, it is not sensible to examine the instantaneous eigenvalue of a trajectory. The next best quantity, therefore, is an eigenvalue averaged over the whole trajectory. The Lyapunov exponent is best defined by measuring the evolution (under a flow) of the tangent manifold

$$
\lambda_{i}=\lim _{t \rightarrow \infty} \frac{1}{t} \ln \left|m_{i}(t)\right|, \quad i=1, \ldots, l
$$

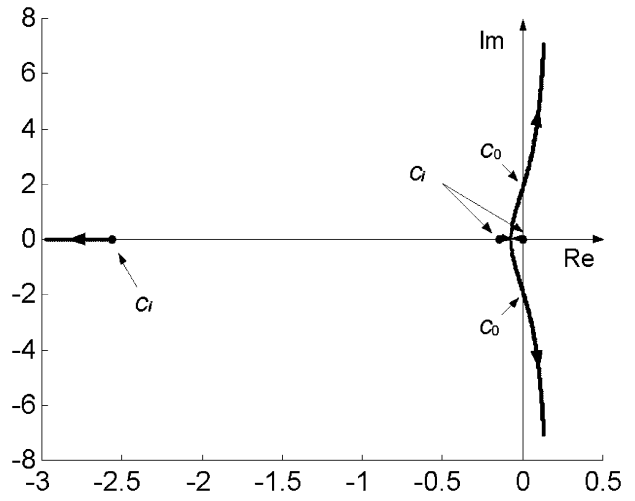

Fig. 3. Locus of eigenvalues via parameter $c$.

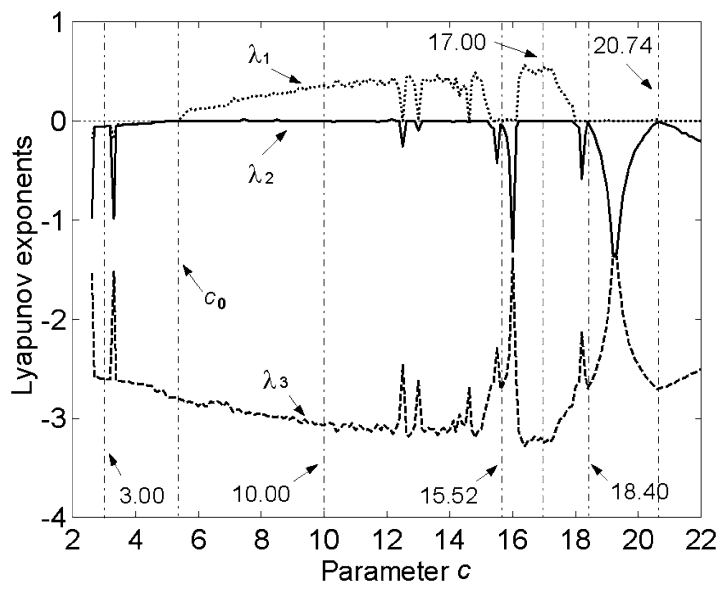

Fig. 4. Lyapunov exponents via parameter $c$.

where $m_{1}(t), \ldots, m_{l}(t)$ is the solution of the system characteristic (14) and $l$ is the system dimension. The Gram-Schmidt orthonormalization algorithm [20] can readily be used to calculate the Lyapunov exponents.

\section{Simulation AND EXPERIMENTAL Results}

To illustrate the strange behavior of the SynRM drive, computer simulations of both waveforms and trajectories are carried out. A three-phase four-pole SynRM drive is used for exemplification, which has the parameters $P=4, L_{d s}=133.3 \mathrm{mH}$, $L_{q s}=25.1 \mathrm{mH}, R_{s}=0.029 \Omega, T_{L}=0 \mathrm{Nm}, J=1.988 \times$ $10^{-3} \mathrm{kgm}^{2}, B=3.513 \times 10^{-3} \mathrm{Nm} / \mathrm{rads}^{-1}, v_{d s}=0 \mathrm{~V}$, and $\omega_{\text {ref }}=0 \mathrm{rad} / \mathrm{s}$. Notice that the values of $v_{d s}, T_{L}$ and $\omega_{\text {ref }}$ are simply chosen for illustrations, and can readily be altered without affecting the final conclusion. Most important, the parameters of this practical motor drive meet the requirements of Hopf bifurcation as described by (21).

The locus of eigenvalues obtained by the equilibrium point pair $\left(\left(c+\sqrt{c^{2}-4 a}\right) / 2, \pm \sqrt{a b}, x_{\mathrm{eq}} y_{\mathrm{eq}} / a\right)$ is depicted in Fig. 3, in which the parameter $c$ starts from $\Delta=0$, namely $c_{i}=$ $2 \sqrt{a}=2.473$. As discussed before, this pair of equilibrium points loses their stability once the parameter $c$ exceeds its critical value. Clearly, at this critical value $c_{0}=5.421$, the locus crosses the imaginary axis and Hopf bifurcation occurs.

Fig. 4 shows the Lyapunov exponents of the SynRM drive system. From these three curves, the underlying system behavior can be easily distinguished by using Table II. 
TABLE III

LYAPUNOV EXPONENTS AT TYPICAL VALUES OF $c$

\begin{tabular}{ccc}
\hline$c$ & Lyapunov Exponents & Type of Attractors \\
\hline 3.00 & $-0.056,-0.056,-2.598$ & Fixed point \\
10.00 & $0.336,0.000,-3.045$ & Chaotic \\
15.52 & $0.000,-0.014,-2.678$ & Limit cycle \\
17.00 & $0.514,0.000,-3.224$ & Chaotic \\
18.40 & $0.000,-0.012,-2.699$ & Limit cycle \\
20.74 & $0.000,-0.010,-2.700$ & Limit cycle \\
\hline
\end{tabular}

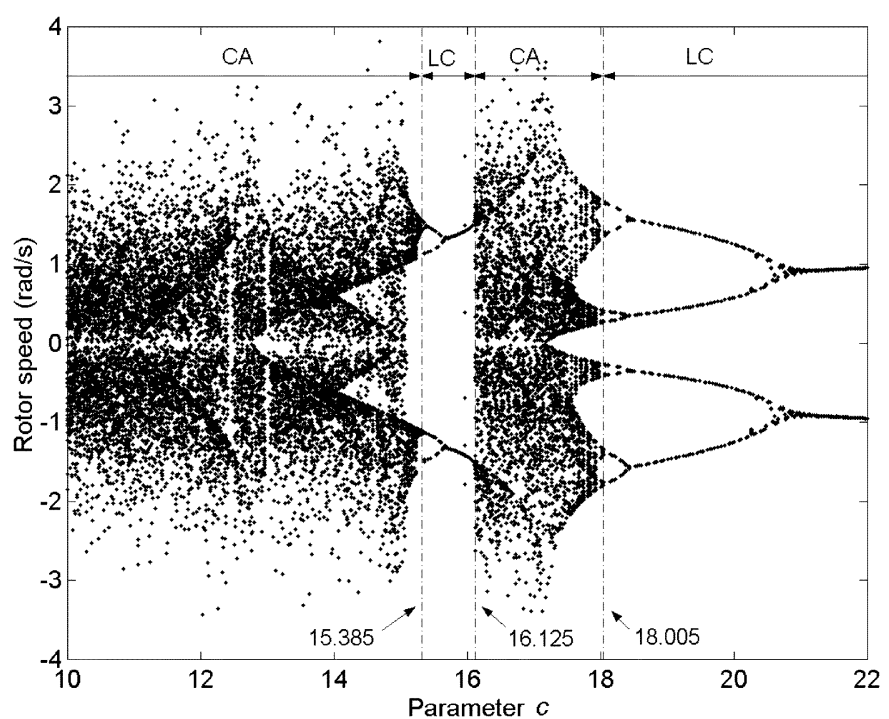

Fig. 5. Speed bifurcation diagram via parameter $c$

With a small value of $c$, the SynRM system has only one stable equilibrium point (fixed point). The corresponding Lyapunov exponents are all negative. Thus, five equilibria occur with the increase of $c$. Once $c$ exceeds its critical value $\left(c_{0}=5.421\right)$, a pair of stable equilibria loses its stability as shown in Fig. 3 and becomes a pair of saddle points. This pair of saddle points causes the SynRM drive demonstrating complex behavior. There are chaotic attractors in the regions of $5.421 \leq c<15.385$ and $16.125 \leq c \leq 17.995$. On the other hand, in the regions of $15.385 \leq c<16.125$ and $c \geq 18.005$, the system exhibits limit cycles. Table III lists the Lyapunov exponents under different typical values of $c$.

Fig. 5 shows the speed bifurcation diagram via $c$. This diagram is obtained by plotting the successive 200 crossing points of the steady-state trajectory with a fixed Poincare section via the parameter $c$. Therefore, the underlying chaotic attractor (CA) or limit cycle (LC) can be easily identified as labeled in the figure. It can be found that this diagram matches the phenomena obtained by Fig. 4 and Table III. Namely, when $15.385 \leq c<16.125$ or $c \geq 18.005$, the system exhibits limit-cycle operations; when $5.421 \leq c<15.385$ or $16.125 \leq c \leq 17.995$, the system offers chaotic operations.

Fig. 6 shows the simulated chaotic waveforms of $i_{d s}, i_{q s}$, and $\omega_{r}$ of the SynRM drive when $c=10$ and the trajectories on the $i_{d s}-i_{q s}, i_{d s}-\omega_{r}$, and $i_{q s}-\omega_{r}$ planes. It can be found that the waveforms offer the well-known chaotic properties, namely random-like and bounded, while the trajectories resemble a butterfly (like the well-known Lorenz attractors).

On the other hand, as illustrated in Fig. 5, the SynRM drive exhibits limit-cycle operation when $c=22$. Fig. 7 depicts the
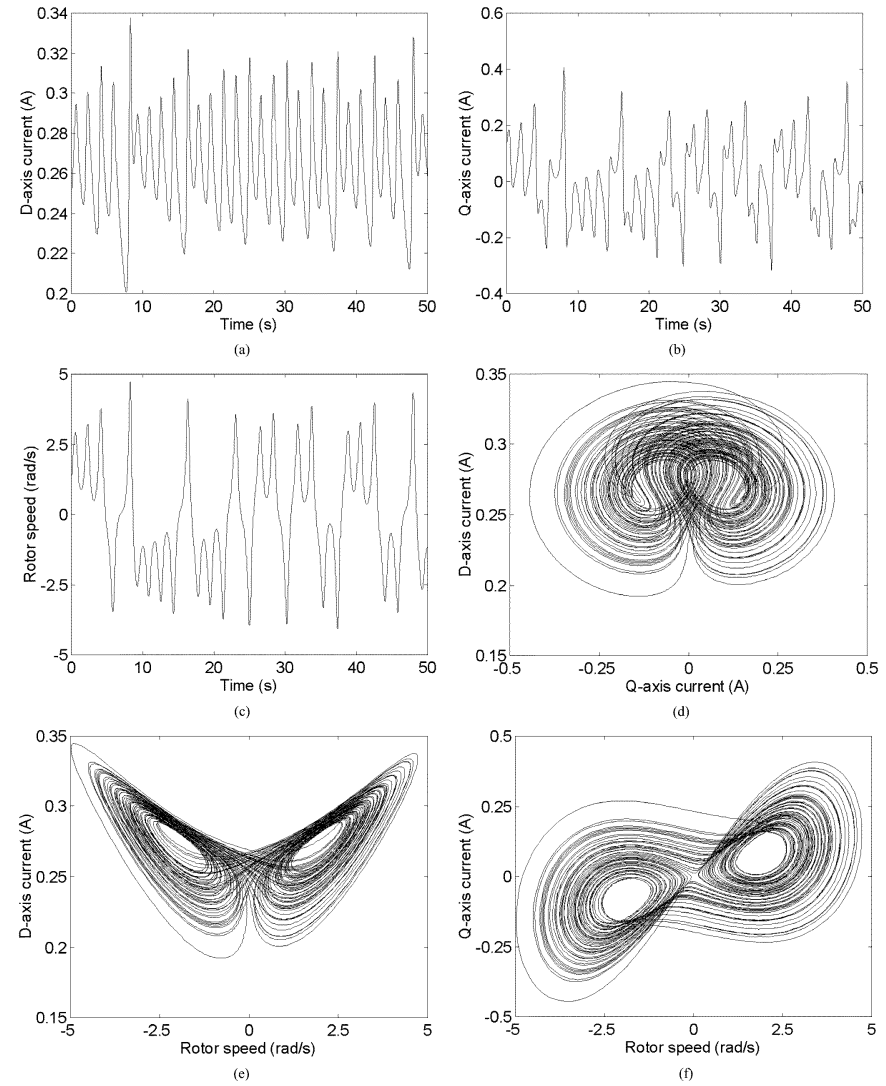

Fig. 6. Simulated chaotic waveforms and trajectories when $c=10$ : (a) $i_{d s}$; (b) $i_{q s}$; (c) $\omega_{r}$; (d) $i_{d s}-i_{q s}$ plane; (e) $i_{d s}-\omega_{r}$ plane; (f) $i_{q s}-\omega_{r}$ plane.
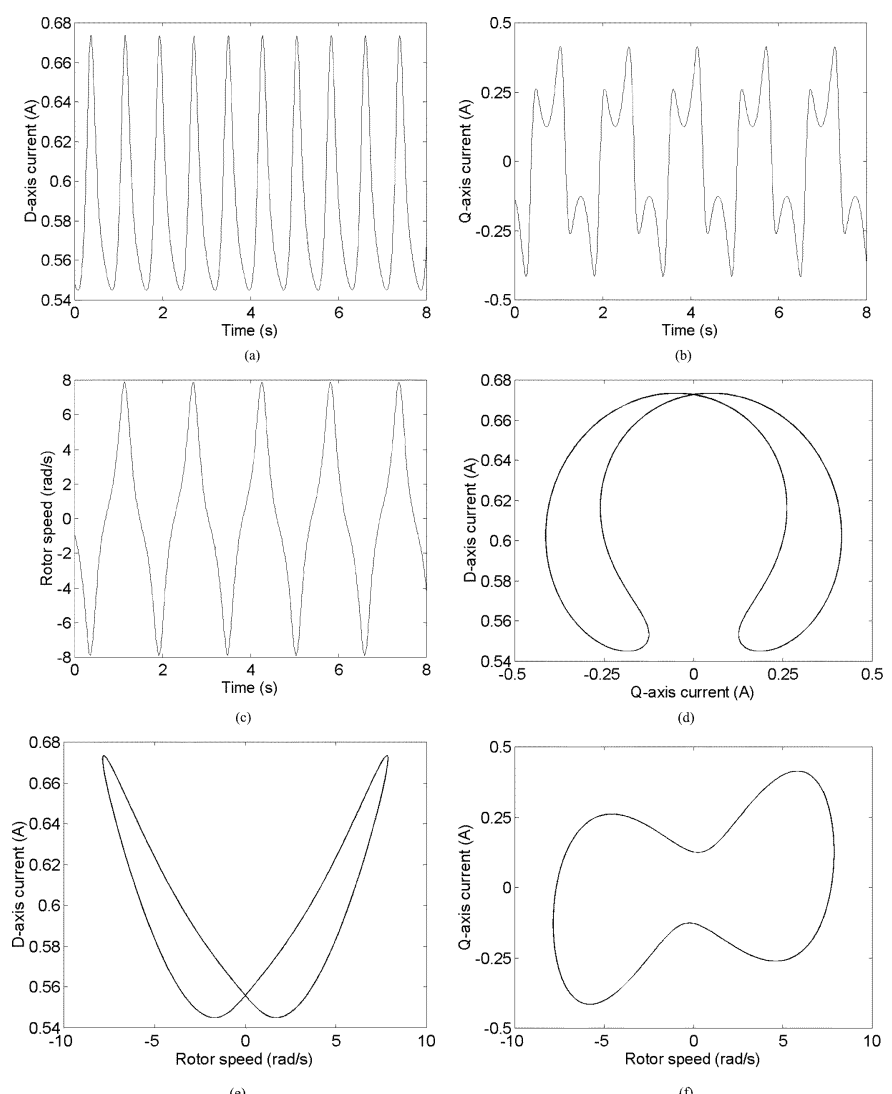

Fig. 7. Simulated periodic waveforms and trajectories when $c=22$ : (a) $i_{d s}$; (b) $i_{q s}$; (c) $\omega_{r}$; (d) $i_{d s}-i_{q s}$ plane; (e) $i_{d s}-\omega_{r}$ plane; (f) $i_{q s}-\omega_{r}$ plane. 

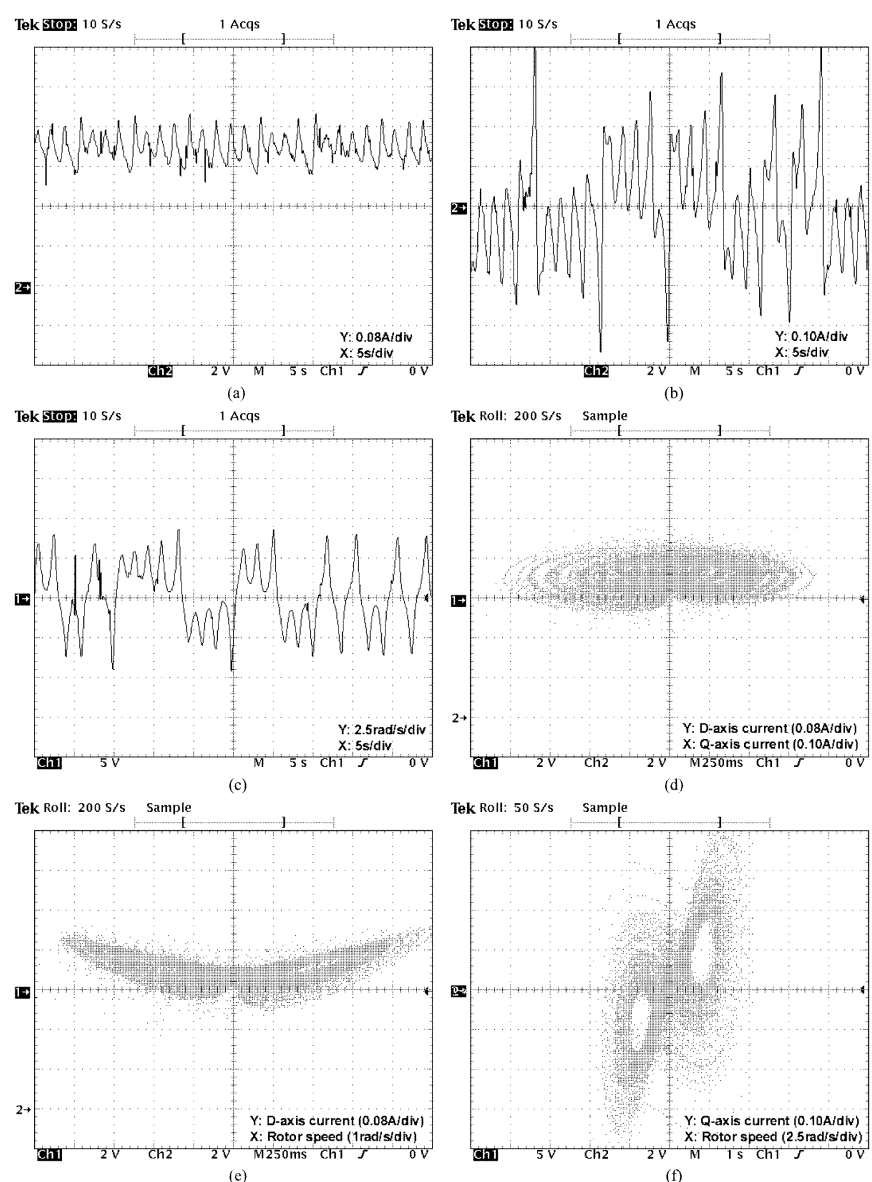

Fig. 8. Measured chaotic waveforms and trajectories when $c=10$ : (a) $i_{d s}$; (b) $i_{q s}$; (c) $\omega_{r}$; (d) $i_{d s}-i_{q s}$ plane; (e) $i_{d s}-\omega_{r}$ plane; (f) $i_{q s}-\omega_{r}$ plane.

corresponding time-domain waveforms of $i_{d s}, i_{q s}$, and $\omega_{r}$, and its trajectories on the $i_{d s}-i_{q s}, i_{d s}-\omega_{r}$, and $i_{q s}-\omega_{r}$ planes. It can easily be observed that both the waveforms and trajectories offer the periodic property.

Experimental results are shown in Figs. 8 and 9. Comparing the periodic waveforms and trajectories shown in Fig. 7 with those in Fig. 9, the measured results match well with the simulation results. On the other hand, comparing the chaotic waveforms shown in Figs. 6 and 8, the simulated and measured patterns can hardly match together because the chaotic pattern is aperiodic and very sensitive to initial conditions. Nevertheless, the random-like and bounded nature can be easily observed from their trajectories. The measured boundary values also match well with the simulated values.

It should be noted that the above analysis regulates the classical concept of SynRM drive operation. Besides the fixed-point operation, the SynRM drive can offer the chaotic operation and limit-cycle operation with the variation of controllable parameters. Although the chaotic operation has not yet been widely used for industrial applications, it is anticipated that the resulting chaotic motion of this SynRM drive is beneficial to surface milling or material mixing.

\section{CONCLUSION}

In this paper, Hopf bifurcation and chaos in a practical SynRM drive have been presented. The key is to analyze the
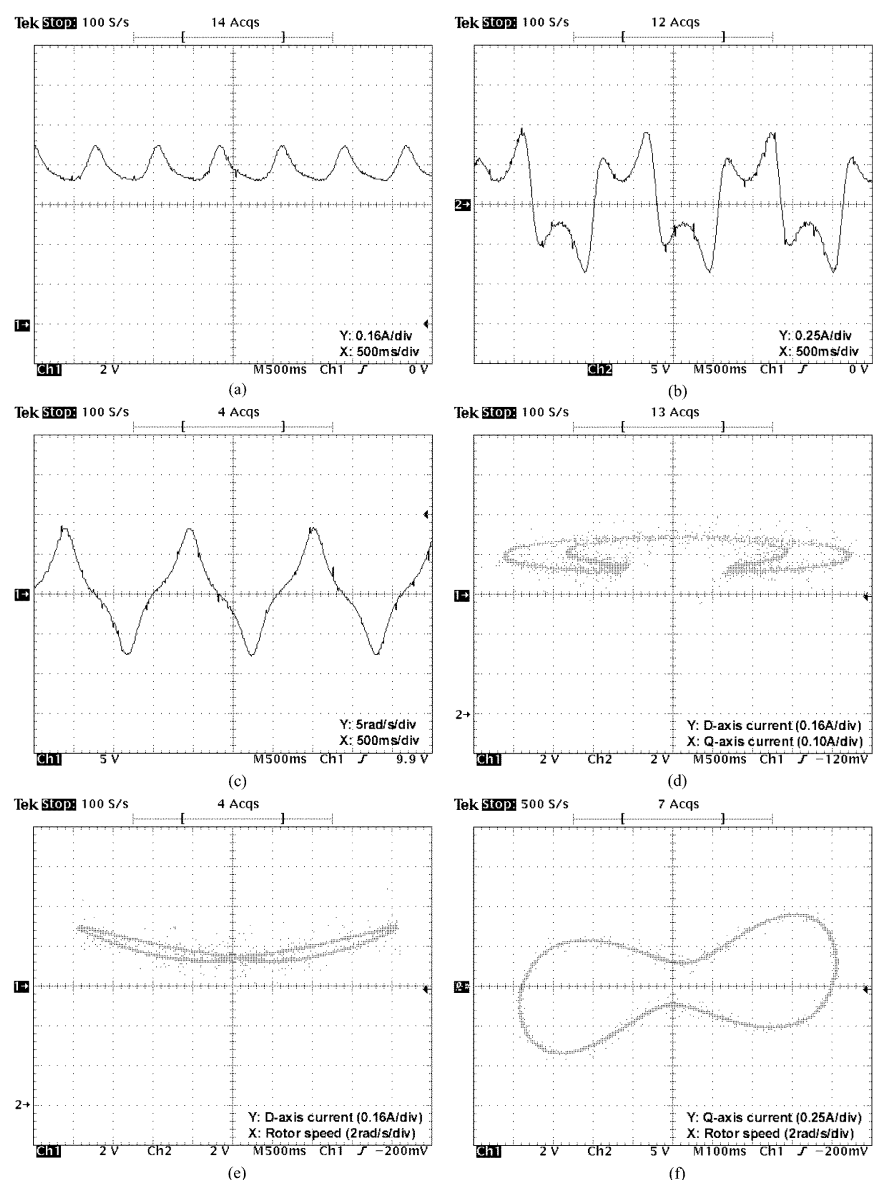

Fig. 9. Measured periodic waveforms and trajectories when $c=22$ : (a) $i_{d s}$; (b) $i_{q s}$; (c) $\omega_{r}$; (d) $i_{d s}-i_{q s}$ plane; (e) $i_{d s}-\omega_{r}$ plane; (f) $i_{q s}-\omega_{r}$ plane.

eigenvalues and Lyapunov exponents of the SynRM drive adopting field-oriented control. Hence, it first identifies that the drive system may offer chaotic operation or limit-cycle operation. Moreover, the proposed analysis is so general that it can readily be extended to other motor drives.

\section{ACKNOWLEDGMENT}

The authors wish to thank the anonymous reviewers for their valuable comments.

\section{REFERENCES}

[1] T. A. Lipo, "Synchronous reluctance machines-A viable alternative for AC drives?," Elect. Mach. Power Syst., vol. 19, no. 6, pp. 659-671, 1991.

[2] L. Xu, X. Xu, T. A. Lipo, and D. W. Novotny, "Vector control of a synchronous reluctance motor including saturation and iron loss," IEEE Trans. Ind. Applicat., vol. 27, pp. 977-985, Sept./Oct. 1991.

[3] R. E. Betz, R. Lagerquist, M. Jovanovic, T. J. E. Miller, and R. H. Middleton, "Control of synchronous reluctance machines," IEEE Trans. Ind. Applicat., vol. 29, pp. 1110-1122, Nov./Dec. 1993.

[4] T. Sharaf-Eldin, M. W. Dunnigan, J. E. Fletcher, and B. W. Williams, "Nonlinear robust control of a vector-controlled synchronous reluctance machine," IEEE Trans. Power Electron., vol. 14, pp. 1111-1121, Nov. 1999.

[5] C. C. Chan and K. T. Chau, "An overview of power electronics in electric vehicles," IEEE Trans. Ind. Electron., vol. 44, pp. 3-13, Feb. 1997.

[6] D. C. Hamill, J. H. B. Deane, and D. J. Jefferies, "Modeling of chaotic dc-dc converters by iterated nonlinear mappings," IEEE Trans. Power Electron., vol. 7, pp. 25-36, Jan. 1992.

[7] E. Fossas and G. Olivar, "Study of chaos in the buck converter," IEEE Trans. Circuits Syst. I, vol. 43, pp. 13-25, Jan. 1996. 
[8] K. Chakrabarty, G. Poddar, and S. Banerjee, "Bifurcation behavior of the buck converter," IEEE Trans. Power Electron., vol. 11, pp. 439-447, May 1996.

[9] M. D. Bernardo, F. Garofalo, L. Glielmo, and F. Vasca, "Switchings, bifurcations, and chaos in DC/DC converters," IEEE Trans. Circuits Syst. I, vol. 45, pp. 133-141, Feb. 1998.

[10] Z. Suto, I. Nagy, and E. Masada, "Avoiding chaotic processes in current control of AC drive," in Proc. IEEE Power Electron. Specialists Conf., 1998, pp. 255-261.

[11] N. Hemati, "Strange attractors in brushless DC motors," IEEE Trans. Circuits Syst. I, vol. 41, pp. 40-45, Jan. 1994.

[12] Z. Li, J. B. Park, Y. H. Joo, B. Zhang, and G. Chen, "Bifurcations and chaos in a permanent-magnet synchronous motor," IEEE Trans. Circuits Syst. I, vol. 49, pp. 383-387, Mar. 2002.

[13] J. H. Chen, K. T. Chau, S. M. Siu, and C. C. Chan, "Experimental stabilization of chaos in a voltage-mode DC drive system," IEEE Trans. Circuits Syst. I, vol. 47, pp. 1093-1095, July 2000.

[14] J. H. Chen, K. T. Chau, and C. C. Chan, "Analysis of chaos in currentmode-controlled DC drive systems," IEEE Trans. Ind. Electron., vol. 47, pp. 67-76, Feb. 2000

[15] K. T. Chau, J. H. Chen, C. C. Chan, and Q. Jiang, "Subharmonics and chaos in switched reluctance motor drives," IEEE Trans. Energy Conversion, vol. 17, pp. 73-78, Mar. 2002.

[16] T. Matsuo and T. A. Lipo, "Field oriented control of synchronous reluctance machine," in Proc. IEEE Power Electron. Specialists Conf., 1993, pp. 425-431.

[17] A. E. Fitzgerald, C. Kingsley Jr., and S. D. Umans, Electric Machinery. New York: McGraw-Hill, 1991.

[18] K. T. Alligood, T. D. Sauer, and J. A. Yorke, Chaos: An Introduction to Dynamical Systems. New York: Springer-Verlag, 1996.

[19] R. C. Hilborn, Chaos and Nonlinear Dynamics: An Introduction for Scientists and Engineers. London, U.K.: Oxford Univ. Press, 1994.
[20] T. S. Parker and L. O. Chua, Practical Numerical Algorithms for Chaotic Systems. New York: Springer-Verlag, 1989.

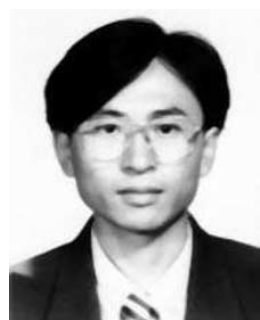

Y. Gao (S'01) was born in Hubei, China, in 1976. $\mathrm{He}$ received the B.Sc. degree in engineering from Shanghai Jiaotong University, Shanghai, China, in 1998, and the M.Phil. degree in electrical and electronic engineering from The Hong Kong University of Science and Technology, Hong Kong, in 2000. $\mathrm{He}$ is currently pursuing the Ph.D. degree at The University of Hong Kong, Hong Kong.

His research interests include motor drives, power electronics, nonlinear system, and chaos application.

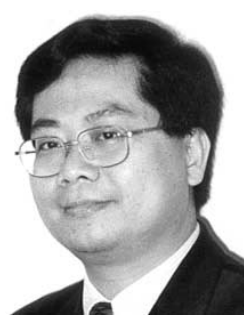

K. T. Chau (M'89-SM'04) received the B.Sc. degree in engineering with first class honors and the M.Phil. and Ph.D. degrees in electrical and electronic engineering from The University of Hong Kong, Hong Kong, China.

Currently, he is an Associate Professor at The University of Hong Kong. His teaching and research interests focus on three main areas-power converters, machines and drives, and electric vehicles. He has published many refereed technical papers and industrial reports in these areas.

Dr. Chau has served as a chair and organizing committee member for many international conferences. 